To be submitted to

IEEE Transactions on Plasma Science

Special Issue on Imaging in Plasma Physics

\title{
Imaging the separation of cathodic arc plasma and macroparticles in curved magnetic filters
}

\author{
André Anders \\ Lawrence Berkeley National Laboratory, University of California \\ 1 Cyclotron Road, Mailstop 53 \\ Berkeley, California 94720, USA.
}

Tel. (510) 486-6745

Fax (510) 486-4374

Aanders@1bl.gov

August 15, 2001

rev. September 26, 2001

This work was supported through CRADA BG98-084(01) by the ER-LTR Program of the U.S. Department of Energy, under Contract No. DE-AC03-76SF00098, and by $\mathrm{CVC}$, Inc., the CRADA Industrial Partner. 


\title{
Imaging the separation of cathodic arc plasma and macroparticles in curved magnetic filters \\ André Anders \\ Lawrence Berkeley National Laboratory, University of California \\ 1 Cyclotron Road, Mailstop 53, Berkeley, California 94720, USA.
}

\begin{abstract}
Cathodic vacuum arc plasmas can be used to produce well adherent, dense, highquality films and coatings provided macroparticles are removed from the plasma. Both plasma and macroparticles are produced at cathode spots, and in most cases both can easily be observed because they emit light. The nature of this light emission is briefly discussed. Using open, curved solenoids as magnetic filters, one can readily optically record the separation of plasma and macroparticles by the filter. Imaging can be used as a powerful tool to help developing macroparticle filters and optimizing their design.
\end{abstract}

Keywords: imaging, cathodic vacuum arc, plasma filter, macroparticles 
Cathodic vacuum arc plasmas can be used to produce well adherent, dense, highquality films and coatings provided macroparticles are removed from the plasma. The arc discharge is concentrated at the cathode surface in cathode spots, micrometer-size locations of high current and power density. While the spot represents a small volume of metal plasma of a few electron-volts temperature, the cathode is globally near room temperature. There exists a thin transition layer of molten cathode material between spot plasma and cathode bulk that is subject to high plasma pressure which surpasses atmospheric pressure by orders of magnitudes [1]. This transition layer is the origin of hot ejected droplets and debris which is often referred to as macroparticles, emphasizing their massive nature compared to electrons and ions.

The temperature of macroparticles at ejection is between the melting and boiling temperature of the cathode material. For high-melting point materials such as tungsten and molybdenum, the macroparticle temperature is high enough that the thermal radiation emitted can be readily seen by the human eye and recorded by a camera. For materials of low melting temperature, the recording camera must be sensitive in the near infrared spectral region.

The plasma at the cathode spot is very dense and therefore subject to many collisions between electrons and heavy particles (atoms and ions). When the plasma expands into the vacuum or low-pressure environment ambient, the collision rates drop significantly. As a result, the spot and near-spot plasma is very bright but the expanded plasma is not. Because the intensity of light emitted depends on the density of plasma and the excitation conditions, one cannot directly identify light intensity with plasma density. For large distances from the spot (e.g. of order millimeters), the rate of inelastic collisions becomes very small, the plasma is in thermodynamic non-equilibrium. The ionization and recombination rates are very small so that the plasma is said to have "frozen" states of ionization (see [2] and references therein). Not only the light intensity but also the spectrum depends on the distance from the spot. While continuum radiation due to free-free transitions and radiative recombination is observed at the cathode spot, bound-bound transitions cause line radiation at larger distances from the spot. Only a fraction of radiation is in the visible because most ion spectral lines are in the UV spectral region, especially the lines of multiply charged ions.

Many approaches have been proposed and tested for the elimination of macroparticles from cathodic vacuum arc plasmas. Most successful are curved magnetic filters, originally introduced by Aksenov and co-workers in the late 1970s [3]. Macroparticle generation, transport and magnetic filters have been reviewed by a number of researchers, see e.g. [4-7]. In a recent work [8] we have brought forward arguments why open, compact filters [9] have excellent performance in terms of plasma throughput and macroparticle removal capability. Up to about $40 \%$ of ions are transmitted in such short, high-field filters. The latest in a series of development steps is a very compact open S-filter that is twisted out of plane, a "twist filter" for short, similar in geometry to a much larger, closed, out-of-plane filter [10]. Figure 1 shows such a compact open twist filter. In this embodiment, the filter coil consists of two $90^{\circ}$-curved filter segments twisted by $45^{\circ}$. The coil is made of flat copper "wire" such as to maximize the likelihood that a macroparticle can leave the plasma volume while providing good electrical conductivity and mechanical integrity. The performance, use, and other details have been published elsewhere [8]. 
Because it is difficult to visualize the separation of plasma and macroparticles with a twisted S-filter, we used an open, non-twisted S-filter to produce numerous insightful pictures. Figure 2 shows one of these pictures. One can clearly see that (i) macroparticles move along straight trajectories, (ii) if a macroparticle hits a surface, such as the turn of a coil, it is elastically reflected, (iii) macroparticles leave the plasma volume through openings between the turns of the field coil, (iv) a large fraction of the plasma is guided by the magnetic filter, and actually well focused within the filter, (v) the plasma is very bright near the source and but much less bright in and after the filter. With calibration using deposition probes, plasma imaging can be used to approximately quantify the plasma, although, as mentioned above, intensity is not proportional to density due to locally different excitation conditions. Pictures like the one of Fig. 2 can be quickly obtained using cameras, and their evaluation is possible with imaging software. By gating the exposure time of a CCD camera, the trajectories of macroparticles appear as finite line segments that can be used to determine the velocity distribution of macroparticles [11]. Imaging tools like this help to optimize macroparticle filters for high-tech applications such as the deposition of ultrathin diamond-like carbon films on read-write heads in the magnetic storage industry [12].

I like to thank Robert MacGill who did most of the design work of the twist filter, furthermore Tom McVeigh, Tom Miller, Ian Brown, Othon Monteiro, Marcela Bilek, Francis Ryan, and Dave Baldwin who all have contributed to this work in various ways. This work was supported through CRADA BG98-084(01) by the ER-LTR Program of the U.S. Department of Energy, under Contract No. DE-AC03-76SF00098, and by CVC, Inc., the CRADA Industrial Partner. 


\section{References}

[1] G. A. Mesyats, Cathode Phenomena in a Vacuum Discharge: The Breakdown, the Spark, and the Arc. Moscow, Russia: Nauka, 2000.

[2] A. Anders, "Plasma fluctuations, local partial Saha equilibrium, and the broadening of vacuum-arc ion charge state distributions," IEEE Trans. Plasma Sci., vol. 27, pp. 1060-1067, 1999.

[3] I. I. Aksenov, V. A. Belous, and V. G. Padalka, "Apparatus to rid the plasma of a vacuum arc of macroparticles," Instrum. Exp. Tech., vol. 21, pp. 1416-1418, 1978.

[4] D. M. Sanders, D. B. Boercker, and S. Falabella, "Coatings technology based on the vacuum arc - a review," IEEE Trans. Plasma Sci., vol. 18, pp. 883-894, 1990.

[5] R. L. Boxman, V. Zhitomirsky, B. Alterkop, E. Gidalevitch, I. Beilis, M. Keidar, and S. Goldsmith, "Recent progress in filtered vacuum arc deposition," Surf. \& Coat. Technol, vol. 86-87, pp. 243-253, 1996.

[6] R. L. Boxman and S. Goldsmith, "Macroparticle contamination in cathodic arc coatings: Generation, transport and control," Surf. \& Coat. Technol., vol. 52, pp. 39$50,1992$.

[7] A. Anders, "Approaches to rid cathodic arc plasma of macro- and nanoparticles: a review," Surf. \& Coat. Technol., vol. 120-121, pp. 319-330, 1999.

[8] A. Anders and R. A. MacGill, "Twist Filter for the removal of macroparticles from cathodic arc plasmas," Surf. \& Coat. Technol., vol. 133-134, pp. 96-100, 2000.

[9] J. Koskinen, A. Anttila, and J.-P. Hirvonen, "Diamond-like carbon coatings by arcdischarge methods," Surf. Coat. Technol., vol. 47, pp. 180-187, 1991.

[10] X. Shi, B. K. Tay, H. S. Tan, E. Liu, J. Shi, L. K. Cheah, and X.Jin, "Transport of vacuum arc plasma through an off-plane double bend filtering duct," Thin Solid Films, vol. 345, pp. 1-6, 1999.

[11] T. Schülke and A. Anders, "Velocity distribution of carbon macroparticles generated by pulsed vacuum arcs," Plasma Sources Sci. Technol., vol. 8, pp. 567-571, 1999.

[12] A. Anders, W. Fong, A. Kulkarni, F. R. Ryan, and C. S. Bhatia, "Ultrathin diamond-like carbon films deposited by filtered carbon vacuum arcs," IEEE Trans. Plasma Sci., vol. 29, no.5, 2001. 


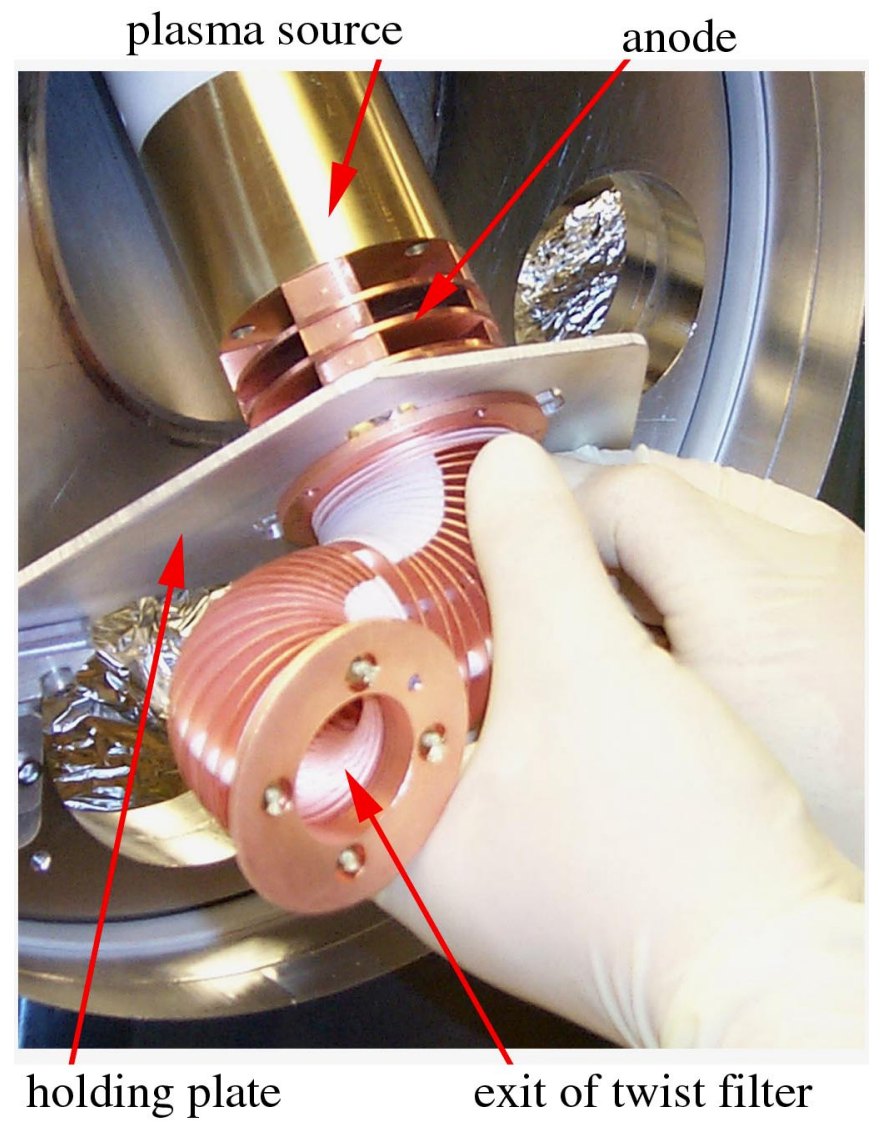

Figure 1 Photograph of a twist filter during mounting. The hands (in gloves) show the small size of the filter. 


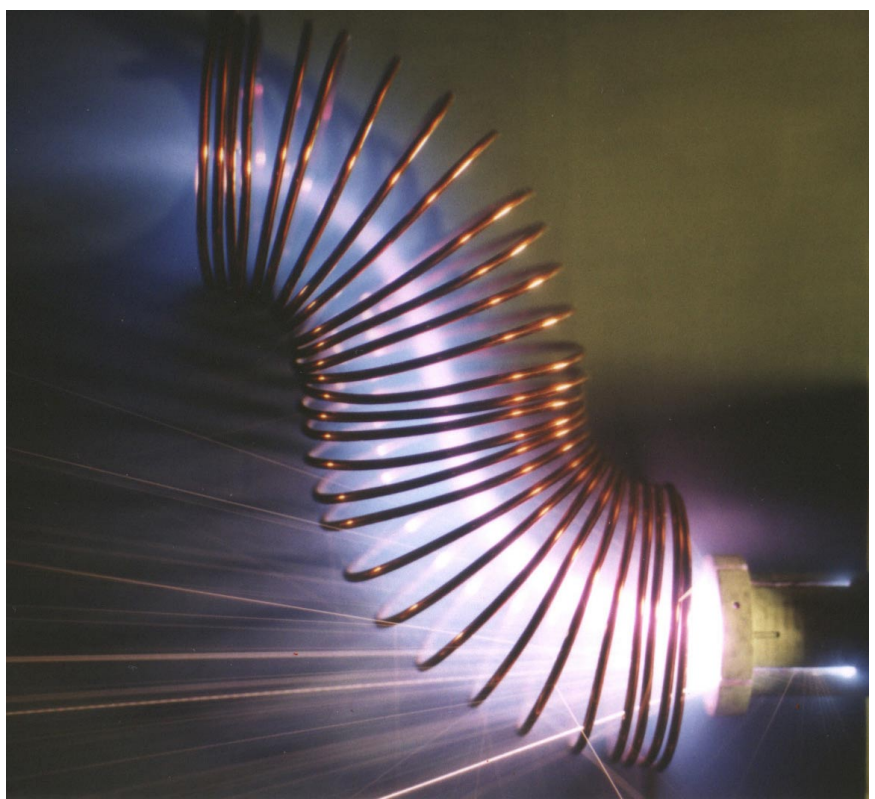

Figure 2 Open-shutter photograph showing the separation of macroparticles and cathodic arc plasma in an open S-filter. Graphite cathode, vacuum base pressure $10^{-6}$ Torr, arc current and coil current in series, both $1 \mathrm{kA}$ for $500 \mu \mathrm{s}$ arc pulse duration. The inner diameter of the turns of the filter coil is $55 \mathrm{~mm}$. 